\title{
AROUND JENSEN'S INEQUALITY FOR STRONGLY CONVEX FUNCTIONS
}

\author{
HAMID REZA MORADI ${ }^{1}$, MOHSEN ERFANIAN OMIDVAR ${ }^{2}$, MUHAMMAD ADIL KHAN ${ }^{3}$ AND \\ KAZIMIERZ NIKODEM ${ }^{4}$
}

\begin{abstract}
In this paper we use basic properties of strongly convex functions to obtain new inequalities including Jensen type and Jensen-Mercer type inequalities. Applications for special means are pointed out as well. We also give a Jensen's operator inequality for strongly convex functions. As a corollary, we improve the Hölder-McCarthy inequality under suitable conditions. More precisely we show that if $\operatorname{Sp}(A) \subset(1, \infty)$, then

$$
\langle A x, x\rangle^{r} \leq\left\langle A^{r} x, x\right\rangle-\frac{r^{2}-r}{2}\left(\left\langle A^{2} x, x\right\rangle-\langle A x, x\rangle^{2}\right), \quad r \geq 2
$$
\end{abstract}

and if $S p(A) \subset(0,1)$, then

$$
\left\langle A^{r} x, x\right\rangle \leq\langle A x, x\rangle^{r}+\frac{r-r^{2}}{2}\left(\langle A x, x\rangle^{2}-\left\langle A^{2} x, x\right\rangle\right), \quad 0<r<1
$$

for each positive operator $A$ and $x \in \mathcal{H}$ with $\|x\|=1$.

\section{Introduction and Preliminaries}

Let $I \subset \mathbb{R}$ be an interval and $c$ be a positive number. Following Polyak [20], a function $f: I \rightarrow \mathbb{R}$ is called strongly convex with modulus $c$ if

$$
f(\lambda x+(1-\lambda) y) \leq \lambda f(x)+(1-\lambda) f(y)-c \lambda(1-\lambda)(x-y)^{2},
$$

for all $x, y \in I$ and $\lambda \in[0,1]$. Obviously, every strongly convex function is convex. Observe also that, for instance, affine functions are not strongly convex. Since strong convexity is strengthening the notion of convexity, some properties of strongly convex functions are just stronger versions of known properties of convex functions. For instance, a function $f: I \rightarrow \mathbb{R}$ is strongly convex with modulus $c$ if and only if for every $x_{0} \in \stackrel{o}{I}$ (the interior of $I$ ) there exists a number $l \in \mathbb{R}$ such that

$$
c\left(x-x_{0}\right)^{2}+l\left(x-x_{0}\right)+f\left(x_{0}\right) \leq f(x), \quad x \in I .
$$

2010 Mathematics Subject Classification. Primary 47A63, 26B25. Secondary 46L05.

Key words and phrases. Strongly convex functions, operator inequality, Jensen's inequality. 
In other words, $f$ has a quadratic support at $x_{0}$. For differentiable functions $f, f$ is strongly convex with modulus $c$ if and only if

$$
\left(f^{\prime}(x)-f^{\prime}(y)\right)(x-y) \geq 2 c(x-y)^{2},
$$

for each $x, y \in I$. We recommend the book [21] and the articles [18, 19] for more details on strongly convex functions.

A basic result concerning the convex functions is Jensen's inequality. Its formal statement is as follows: If $f$ is a convex function on an interval $[m, M]$, then

$$
f\left(\sum_{i=1}^{n} p_{i} x_{i}\right) \leq \sum_{i=1}^{n} p_{i} f\left(x_{i}\right)
$$

for all $x_{i} \in[m, M]$ and all $p_{i} \in[0,1](i=1, \ldots, n)$ with $\sum_{i=1}^{n} p_{i}=1$.

There are several inequalities which are special kinds of this inequality. So, many mathematicians paid their attention to get generalizations and reformulations of this inequality.

Consider a real valued function $f$ defined on an interval $I, x_{1}, \ldots, x_{n} \in I$ and $p_{1}, \ldots, p_{n} \in$ $[0,1]$ with $\sum_{i=1}^{n} p_{i}=1$. The Jensen functional is defined by

$$
\mathcal{J}_{n}(f, \mathbf{x}, \mathbf{p})=\sum_{i=1}^{n} p_{i} f\left(x_{i}\right)-f\left(\sum_{i=1}^{n} p_{i} x_{i}\right) .
$$

According to $\left[2\right.$, Theorem 1], if $\mathbf{x}=\left(x_{1}, \ldots, x_{n}\right) \in I^{n}, \mathbf{p}=\left(p_{1}, \ldots, p_{n}\right), \mathbf{q}=\left(q_{1}, \ldots, q_{n}\right)$ are non-negative $n$-tuples satisfying $\sum_{i=1}^{n} p_{i}=1, \sum_{i=1}^{n} q_{i}=1, q_{i}>0, i=1, \ldots, n$, then

$$
\min _{1 \leq i \leq n}\left\{\frac{p_{i}}{q_{i}}\right\} \mathcal{J}_{n}(f, \mathbf{x}, \mathbf{q}) \leq \mathcal{J}_{n}(f, \mathbf{x}, \mathbf{p}) \leq \max _{1 \leq i \leq n}\left\{\frac{p_{i}}{q_{i}}\right\} \mathcal{J}_{n}(f, \mathbf{x}, \mathbf{q})
$$

For more results concerning Jensen's functional the reader is referred to [12, 22].

In paper [11, Theorem 1.2] Mercer proved the following variant of Jensen's inequality, to which we will refer as to the Jensen-Mercer's inequality. If $f$ is a convex function on $[m, M]$, then

$$
f\left(M+m-\sum_{i=1}^{n} p_{i} x_{i}\right) \leq f(M)+f(m)-\sum_{i=1}^{n} p_{i} f\left(x_{i}\right),
$$

for all $x_{i} \in[m, M]$ and all $p_{i} \in[0,1](i=1, \ldots, n)$ with $\sum_{i=1}^{n} p_{i}=1$. We refer the reader to $[4,7,17]$ as a sample of the extensive use of this inequality in this field.

The principal aim of this article is to derive some results related to the Jensen functional in the framework of strongly convex functions (see Theorem 2.1). We present Jensen-Mercer's inequality for this class of functions (see Theorem 2.2) and give some applications for means 
(Corollary 2.2). In particular some new refinements of Jensen's operator inequality for strongly convex functions are also given (Theorem 3.3 and Theorem 3.4).

\section{On The Jensen Inequality For Strongly Convex Functions}

The following lemma due to Merentes and Nikodem [15, Theorem 4] is the starting point for our discussion.

Lemma 2.1. If $f: I \rightarrow \mathbb{R}$ is strongly convex with modulus $c$, then

$$
c \sum_{i=1}^{n} p_{i}\left(x_{i}-\bar{x}\right)^{2} \leq \mathcal{J}_{n}(f, \mathbf{x}, \mathbf{p}),
$$

for all $x_{1}, \ldots, x_{n} \in I, p_{1}, \ldots, p_{n}>0$ with $p_{1}+\ldots+p_{n}=1$ and $\bar{x}=\sum_{i=1}^{n} p_{i} x_{i}$.

In [13, Corollary 3] the following result has been given:

Theorem 2.1. Let $f$ be a strongly convex function with modulus $c, \mathbf{x}=\left(x_{1}, \ldots, x_{n}\right) \in I^{n}, \mathbf{p}=$ $\left(p_{1}, \ldots, p_{n}\right), \mathbf{q}=\left(q_{1}, \ldots, q_{n}\right)$ non-negative $n$-tuples satisfying $\sum_{i=1}^{n} p_{i}=1, \sum_{i=1}^{n} q_{i}=1, q_{i}>0, i=$ $1, \ldots, n$. Then

$$
\begin{aligned}
& m \mathcal{J}_{n}(f, \mathbf{x}, \mathbf{q})+c\left(\sum_{i=1}^{n}\left(p_{i}-m q_{i}\right)\left(x_{i}-\sum_{i=1}^{n} p_{i} x_{i}\right)^{2}+m\left(\sum_{i=1}^{n}\left(p_{i}-q_{i}\right) x_{i}\right)^{2}\right)^{2} \\
& \leq \mathcal{J}_{n}(f, \mathbf{x}, \mathbf{p}) \\
& \leq M \mathcal{J}_{n}(f, \mathbf{x}, \mathbf{q})-c\left(\sum_{i=1}^{n}\left(M q_{i}-p_{i}\right)\left(x_{i}-\sum_{j=1}^{n} q_{j} x_{j}\right)^{2}+\left(\sum_{i=1}^{n}\left(p_{i}-q_{i}\right) x_{i}\right)^{2}\right),
\end{aligned}
$$

where

$$
m:=\min _{1 \leq i \leq n}\left\{\frac{p_{i}}{q_{i}}\right\}, \quad M:=\max _{1 \leq i \leq n}\left\{\frac{p_{i}}{q_{i}}\right\} .
$$

Remark 2.1. Notice that Lemma 2.1 also holds for functions defined on open convex subsets of an inner product space (cf. [18, Theorem 2]). Therefore, Theorem 2.1 can be also formulated and proved in such more general settings.

An interesting corollary can be deduced at this stage. We restrict ourselves to the case when $n=2$.

Remark 2.2. According to Hiriart-Urruty and Lemaréchal [3, Prop 1.1.2], the function $f$ : $I \rightarrow \mathbb{R}$ is strongly convex with modulus $c$ if and only if the function $g: I \rightarrow \mathbb{R}$ defined by $g(x)=f(x)-c x^{2}$ is convex. Hence the function $f:(0,1] \rightarrow[0, \infty), f(x)=-\ln x$ is strongly 
convex with modulus $c=\frac{1}{2}$. Taking $p_{1}=\lambda, p_{2}=1-\lambda, q_{1}=\mu, q_{2}=1-\mu$ with $\lambda, \mu \in$ $[0,1], x_{1}=a, x_{2}=b$ and taking into account that $m=\min \left\{\frac{\lambda}{\mu}, \frac{1-\lambda}{1-\mu}\right\}$ and $M=\max \left\{\frac{\lambda}{\mu}, \frac{1-\lambda}{1-\mu}\right\}$, simple algebraic manipulations show that

$$
\begin{aligned}
& \left(\frac{\mu a+(1-\mu) b}{a^{\mu} b^{1-\mu}}\right)^{m} \\
& \quad \times \exp \left(\frac{(b-a)^{2}}{2}\left((\lambda-m \mu)(\lambda-1)^{2}+\lambda^{2}((1-\lambda)-m(1-\mu))+m(\mu-\lambda)^{2}\right)\right) \\
& \leq \frac{\lambda a+(1-\lambda) b}{a^{\lambda} b^{1-\lambda}} \\
& \left.\leq \quad \frac{\mu a+(1-\mu) b}{a^{\mu} b^{1-\mu}}\right)^{M} \\
& \quad \times \frac{1}{\exp \left(\frac{(b-a)^{2}}{2}\left((M \mu-\lambda)(\mu-1)^{2}+\mu^{2}(M(1-\mu)-(1-\lambda))+(\mu-\lambda)^{2}\right)\right)} .
\end{aligned}
$$

Remark 2.2 admits the following important special case.

Corollary 2.1. Let $a, b \in(0,1]$, then

$$
\begin{aligned}
& K^{r}\left(\frac{a}{b}\right) \\
& \quad \times \exp \left(\frac{(b-a)^{2}}{2}\left((\lambda-r)(\lambda-1)^{2}+\lambda^{2}((1-\lambda)-r)+\frac{r}{2}(1-2 \lambda)^{2}\right)\right) \\
& \leq \frac{\lambda a+(1-\lambda) b}{a^{\lambda} b^{1-\lambda}} \\
& \leq K^{R}\left(\frac{a}{b}\right) \\
& \quad \times \frac{1}{\exp \left(\frac{(b-a)^{2}}{8}\left((R-\lambda)+(R-(1-\lambda))+(1-2 \lambda)^{2}\right)\right)},
\end{aligned}
$$

where $r=\min \{\lambda, 1-\lambda\}, R=\max \{\lambda, 1-\lambda\}, \lambda \in[0,1]$ and $K\left(\frac{a}{b}\right)=\frac{(a+b)^{2}}{4 a b}$ is the Kantorovich constant.

Proof. The result follows from Remark 2.2 by taking $\mu=\frac{1}{2}$.

Remark 2.3. The following multiplicative refinement and reverse of the Young inequality in terms of Kantorovich's constant holds:

$$
K^{r}\left(\frac{a}{b}\right) a^{\lambda} b^{1-\lambda} \leq \lambda a+(1-\lambda) b \leq K^{R}\left(\frac{a}{b}\right) a^{\lambda} b^{1-\lambda}
$$

where $a, b>0, \lambda \in[0,1], r=\min \{\lambda, 1-\lambda\}$ and $R=\max \{\lambda, 1-\lambda\}$. 
The first inequality in (2.2) was obtained by Zou et al. in [23, Corollary 3] while the second one by Liao et al. [6, Corollary 2.2].

Since $\exp (x) \geq 1$ for $x \geq 0$, Corollary 2.1 essentially gives a refinement of the inequalities in (2.2).

The key role in our proof for Theorem 2.2 will be played by the following lemma.

Lemma 2.2. If $f: I \rightarrow \mathbb{R}$ is strongly convex with modulus $c$, then

$$
f\left(x_{1}+x_{n}-x_{i}\right) \leq f\left(x_{1}\right)+f\left(x_{n}\right)-f\left(x_{i}\right)-2 c \lambda_{i}\left(1-\lambda_{i}\right)\left(x_{1}-x_{n}\right)^{2},
$$

where $\lambda_{i} \in[0,1], x_{1}=\min _{1 \leq i \leq n} x_{i}, x_{n}=\max _{1 \leq i \leq n} x_{i}$ and $x_{i} \in I$.

Proof. We use the strategy of Mercer [11, Lemma 1.3]. Let $y_{i}=x_{1}+x_{n}-x_{i}, i=1,2, \ldots, n$. We may write $x_{i}=\lambda_{i} x_{1}+\left(1-\lambda_{i}\right) x_{n}$ and $y_{i}=\left(1-\lambda_{i}\right) x_{1}+\lambda_{i} x_{n}$ where $\lambda_{i} \in[0,1]$.

Now, using simple calculations, we obtain

$$
\begin{aligned}
f\left(y_{i}\right) & =f\left(\left(1-\lambda_{i}\right) x_{1}+\lambda_{i} x_{n}\right) \\
& \leq\left(1-\lambda_{i}\right) f\left(x_{1}\right)+\lambda_{i} f\left(x_{n}\right)-c \lambda_{i}\left(1-\lambda_{i}\right)\left(x_{1}-x_{n}\right)^{2} \quad(\text { by }(1.1)) \\
& =f\left(x_{1}\right)+f\left(x_{n}\right)-\left(\lambda_{i} f\left(x_{1}\right)+\left(1-\lambda_{i}\right) f\left(x_{n}\right)\right)-c \lambda_{i}\left(1-\lambda_{i}\right)\left(x_{1}-x_{n}\right)^{2} \\
& \leq f\left(x_{1}\right)+f\left(x_{n}\right)-f\left(\lambda_{i} x_{1}+\left(1-\lambda_{i}\right) x_{n}\right)-2 c \lambda_{i}\left(1-\lambda_{i}\right)\left(x_{1}-x_{n}\right)^{2} \quad(b y \\
& =f\left(x_{1}\right)+f\left(x_{n}\right)-f\left(x_{i}\right)-2 c \lambda_{i}\left(1-\lambda_{i}\right)\left(x_{1}-x_{n}\right)^{2},
\end{aligned}
$$

which completes the proof.

The Lemma 2.2 follows also from the fact that strongly convex functions are strongly Wrightconvex (see, e.g., [16]).

At this point our aim is to present Jensen-Mercer's inequality for strongly convex functions.

Theorem 2.2. Let $f: I \rightarrow \mathbb{R}$ be a strongly convex with modulus $c$, then

$$
\begin{aligned}
& f\left(x_{1}+x_{n}-\sum_{i=1}^{n} p_{i} x_{i}\right) \\
& \leq f\left(x_{1}\right)+f\left(x_{n}\right)-\sum_{i=1}^{n} p_{i} f\left(x_{i}\right)-c\left(2 \sum_{i=1}^{n} p_{i} \lambda_{i}\left(1-\lambda_{i}\right)\left(x_{1}-x_{n}\right)^{2}+\sum_{i=1}^{n} p_{i}\left(x_{i}-\sum_{i=1}^{n} p_{i} x_{i}\right)^{2}\right)
\end{aligned}
$$

where $\sum_{i=1}^{n} p_{i}=1, \lambda_{i} \in[0,1], x_{1}=\min _{1 \leq i \leq n} x_{i}, x_{n}=\max _{1 \leq i \leq n} x_{i}$ and $x_{i} \in I$. 
Proof. A straightforward computation gives that

$$
\begin{aligned}
& f\left(x_{1}+x_{n}-\sum_{i=1}^{n} p_{i} x_{i}\right) \\
& =f\left(\sum_{i=1}^{n} p_{i}\left(x_{1}+x_{n}-x_{i}\right)\right) \\
& \leq \sum_{i=1}^{n} p_{i} f\left(x_{1}+x_{n}-x_{i}\right)-c \sum_{i=1}^{n} p_{i}\left(x_{i}-\sum_{i=1}^{n} p_{i} x_{i}\right)^{2} \quad(\text { by Lemma 2.1) } \\
& \leq f\left(x_{1}\right)+f\left(x_{n}\right)-\sum_{i=1}^{n} p_{i} f\left(x_{i}\right) \\
& \quad-c\left(2 \sum_{i=1}^{n} p_{i} \lambda_{i}\left(1-\lambda_{i}\right)\left(x_{1}-x_{n}\right)^{2}+\sum_{i=1}^{n} p_{i}\left(x_{i}-\sum_{i=1}^{n} p_{i} x_{i}\right)^{2}\right) \quad \text { (by Lemma 2.2), }
\end{aligned}
$$

as required.

Remark 2.4. Based on Theorem 2.2, we obtain that

$$
\begin{aligned}
& f\left(x_{1}+x_{n}-\sum_{i=1}^{n} p_{i} x_{i}\right) \\
& \leq f\left(x_{1}\right)+f\left(x_{n}\right)-\sum_{i=1}^{n} p_{i} f\left(x_{i}\right)-c\left(2 \sum_{i=1}^{n} p_{i} \lambda_{i}\left(1-\lambda_{i}\right)\left(x_{1}-x_{n}\right)^{2}+\sum_{i=1}^{n} p_{i}\left(x_{i}-\sum_{i=1}^{n} p_{i} x_{i}\right)^{2}\right) \\
& \leq f\left(x_{1}\right)+f\left(x_{n}\right)-\sum_{i=1}^{n} p_{i} f\left(x_{i}\right) .
\end{aligned}
$$

Corollary 2.2. Let us now define

$$
\widetilde{\mathscr{A}}:=x_{1}+x_{n}-\mathscr{A}, \quad \widetilde{\mathscr{G}}:=\frac{x_{1} x_{n}}{\mathscr{G}},
$$

where $\mathscr{A}$ and $\mathscr{G}$ denote the usual arithmetic and geometric means respectively.

As mentioned above, the function $f:(0,1] \rightarrow[0, \infty), f(x)=-\ln x$ is strongly convex with modulus $c=\frac{1}{2}$. From Remark 2.4 we obtain

$$
-\ln \widetilde{\mathscr{A}} \leq-\ln \widetilde{\mathscr{G}}-\frac{1}{2} \mathrm{M} \leq-\ln \widetilde{\mathscr{G}}
$$

where $\mathbf{M}=2 \sum_{i=1}^{n} p_{i} \lambda_{i}\left(1-\lambda_{i}\right)\left(x_{1}-x_{n}\right)^{2}+\sum_{i=1}^{n} p_{i}\left(x_{i}-\sum_{i=1}^{n} p_{i} x_{i}\right)^{2}$. Hence

$$
\widetilde{\mathscr{G}} \leq e^{\frac{1}{2} \mathrm{M} \tilde{\mathscr{G}}} \leq \widetilde{\mathscr{A}}
$$

Remark 2.5. The inequality (2.3) is better than an inequality given in [10, Lemma 1.1]. 


\section{Jensen Operator Inequality For Strongly Convex Functions}

Mond and Pečarić [14] gave an operator extension of the Jensen inequality as follows:

Theorem 3.1. Let $A \in \mathcal{B}(\mathcal{H})$ be a self-adjoint operator with $S p(A) \subset[m, M]$ for some scalars $m<M$. If $f(t)$ is a convex function on $[m, M]$, then

$$
f(\langle A x, x\rangle) \leq\langle f(A) x, x\rangle,
$$

for any unit vector $x \in \mathcal{H}$.

The celebrated Hölder-McCarthy inequality [9] which is a special case of Theorem 3.1 asserts that:

Theorem 3.2. Let $A$ be a positive operator on $\mathcal{H}$. If $x \in \mathcal{H}$ is a unit vector, then

(a) $\langle A x, x\rangle^{r} \leq\left\langle A^{r} x, x\right\rangle$ for all $r>1$.

(b) $\langle A x, x\rangle^{r} \geq\left\langle A^{r} x, x\right\rangle$ for all $0<r<1$.

Here we give a more precise estimation than inequality (3.1) for strongly convex functions with modulus $c$ as follows:

Theorem 3.3. (Jensen operator inequality for strongly convex functions) Let $f: I \rightarrow \mathbb{R}$ be strongly convex with modulus $c$ and differentiable on $\stackrel{o}{I}$. If $A$ is a self-adjoint operator on the Hilbert space $\mathcal{H}$ with $\operatorname{Sp}(A) \subset \stackrel{o}{I}$, then

$$
f(\langle A x, x\rangle) \leq\langle f(A) x, x\rangle-c\left(\left\langle A^{2} x, x\right\rangle-\langle A x, x\rangle^{2}\right),
$$

for each $x \in \mathcal{H}$, with $\|x\|=1$.

Proof. It follows from (1.2) by utilizing functional calculus that

$$
c\left(A^{2}+x_{0}^{2} I-2 x_{0} A\right)+l A-l x_{0} I+f\left(x_{0}\right) I \leq f(A),
$$

which is equivalent to

$$
c\left(\left\langle A^{2} x, x\right\rangle+x_{0}^{2}-2 x_{0}\langle A x, x\rangle\right)+l\langle A x, x\rangle-l x_{0}+f\left(x_{0}\right) \leq\langle f(A) x, x\rangle,
$$

for each $x \in \mathcal{H}$, with $\|x\|=1$.

Now, by applying (3.4) for $x_{0}=\langle A x, x\rangle$, we deduce the desired inequality (3.2).

Remark 3.1. Notice that if $A$ is positive, then the quantity $\left\langle A^{2} x, x\right\rangle-\langle A x, x\rangle^{2}$ is positive. Therefore we have

$$
f(\langle A x, x\rangle) \leq\langle f(A) x, x\rangle-c\left(\left\langle A^{2} x, x\right\rangle-\langle A x, x\rangle^{2}\right) \leq\langle f(A) x, x\rangle .
$$

Corollary 3.1. (Applications for Hölder-McCarthy's inequality) 
- Consider the function $f:(1, \infty) \rightarrow \mathbb{R}, f(x)=x^{r}$ with $r \geq 2$. It can be easily verified that this function is strongly convex with modulus $c=\frac{r^{2}-r}{2}$. Based on this fact, from Theorem 3.3 we obtain

$$
\langle A x, x\rangle^{r} \leq\left\langle A^{r} x, x\right\rangle-\frac{r^{2}-r}{2}\left(\left\langle A^{2} x, x\right\rangle-\langle A x, x\rangle^{2}\right),
$$

for each positive operator $A$ with $S p(A) \subset(1, \infty)$ and $x \in \mathcal{H}$ with $\|x\|=1$.

It is obvious that the inequality (3.5) is a refinement of Theorem 3.2 (a).

- It is readily checked that the function $f:(0,1) \rightarrow \mathbb{R}, f(x)=-x^{r}$ with $0<r<1$ is a strongly convex function with modulus $c=\frac{r-r^{2}}{2}$. Similarly to the above, by using Theorem 3.3 we get

$$
\left\langle A^{r} x, x\right\rangle \leq\langle A x, x\rangle^{r}+\frac{r-r^{2}}{2}\left(\langle A x, x\rangle^{2}-\left\langle A^{2} x, x\right\rangle\right),
$$

for each positive operator $A$ with $S p(A) \subset(0,1)$ and $x \in \mathcal{H}$ with $\|x\|=1$.

Apparently, inequality (3.6) is a refinement of Theorem 3.2 (b).

Now, we draw special attention to the case $f^{\nu}, \nu \in[0,1]$ is strongly convex for which further refinement is possible.

Theorem 3.4. Let $f: I \rightarrow \mathbb{R}$ be non-negative and strongly convex with modulus $c$. If $f^{\nu}$ with $\nu \in[0,1]$ is strongly convex with modulus $c^{\prime}$, then

$$
\begin{aligned}
& f(\langle A x, x\rangle) \\
& \leq f^{1-\nu}(\langle A x, x\rangle) f^{\nu}(\langle A x, x\rangle)+c^{\prime} f^{1-\nu}(\langle A x, x\rangle)\left(\left\langle A^{2} x, x\right\rangle-\langle A x, x\rangle^{2}\right) \\
& \leq f^{1-\nu}(\langle A x, x\rangle)\left\langle f^{\nu}(A) x, x\right\rangle \\
& \leq f^{1-\nu}(\langle A x, x\rangle)\langle f(A) x, x\rangle^{\nu} \\
& \leq(1-\nu) f(\langle A x, x\rangle)+\nu\langle f(A) x, x\rangle \\
& \leq\langle f(A) x, x\rangle-c(1-\nu)\left(\left\langle A^{2} x, x\right\rangle-\langle A x, x\rangle^{2}\right) \\
& \leq\langle f(A) x, x\rangle,
\end{aligned}
$$

for any positive operator $A$ with $S p(A) \subset I$ and unit vector $x \in \mathcal{H}$.

Proof. As we have assumed above $f^{\nu}$ is strongly convex, so (3.2) gives

$$
f^{\nu}(\langle A x, x\rangle) \leq\left\langle f^{\nu}(A) x, x\right\rangle-c^{\prime}\left(\left\langle A^{2} x, x\right\rangle-\langle A x, x\rangle^{2}\right) .
$$


Multiplying both sides by $f^{1-\nu}(\langle A x, x\rangle)$ we infer that

$$
\begin{aligned}
& f(\langle A x, x\rangle) \\
& =f^{1-\nu}(\langle A x, x\rangle) f^{\nu}(\langle A x, x\rangle) \\
& \leq f^{1-\nu}(\langle A x, x\rangle)\left\langle f^{\nu}(A) x, x\right\rangle-c^{\prime} f^{1-\nu}(\langle A x, x\rangle)\left(\left\langle A^{2} x, x\right\rangle-\langle A x, x\rangle^{2}\right) .
\end{aligned}
$$

Rearranging the terms, we obtain

$$
\begin{aligned}
& f(\langle A x, x\rangle) \\
& \leq f^{1-\nu}(\langle A x, x\rangle) f^{\nu}(\langle A x, x\rangle)+c^{\prime} f^{1-\nu}(\langle A x, x\rangle)\left(\left\langle A^{2} x, x\right\rangle-\langle A x, x\rangle^{2}\right) \\
& \leq f^{1-\nu}(\langle A x, x\rangle)\left\langle f^{\nu}(A) x, x\right\rangle .
\end{aligned}
$$

On the other hand, by using Hölder-McCarthy inequality we have

$$
\left\langle f^{\nu}(A) x, x\right\rangle \leq\langle f(A) x, x\rangle^{\nu} .
$$

Multiplying both sides by $f^{1-\nu}(\langle A x, x\rangle)$ we obtain

$$
\begin{aligned}
f^{1-\nu}(\langle A x, x\rangle)\left\langle f^{\nu}(A) x, x\right\rangle & \leq f^{1-\nu}(\langle A x, x\rangle)\langle f(A) x, x\rangle^{\nu} \\
& \leq(1-\nu) f(\langle A x, x\rangle)+\nu\langle f(A) x, x\rangle \quad \text { (by Young's inequality) } \\
& \leq\langle f(A) x, x\rangle-c(1-\nu)\left(\left\langle A^{2} x, x\right\rangle-\langle A x, x\rangle^{2}\right) \quad \text { (by (3.2)). }
\end{aligned}
$$

Combining (3.8) and (3.9) yields the desired result (3.7).

By (1.3) and in a manner similar to the proof of Theorem 3.3, we have the following additive reverse:

Theorem 3.5. Let $f: I \rightarrow \mathbb{R}$ be strongly convex with modulus $c$ and differentiable on $\stackrel{\circ}{I}$ whose derivative $f^{\prime}$ is continuous on $\stackrel{\circ}{I}$. If $A$ is a self-adjoint operator on the Hilbert space $\mathcal{H}$ with $\operatorname{Sp}(A) \subset \stackrel{\circ}{I}$, then

$$
\left\langle A^{2} x, x\right\rangle-\langle A x, x\rangle^{2} \leq \frac{1}{2 c}\left(\left\langle f^{\prime}(A) A x, x\right\rangle-\langle A x, x\rangle\left\langle f^{\prime}(A) x, x\right\rangle\right),
$$

for each $x \in \mathcal{H}$, with $\|x\|=1$.

\section{Further Generalization}

By replacing $c(x-y)^{2}$ with a non-negative real valued function $F(x-y)$, we can define F-strongly convex functions as follows:

$$
f(\lambda x+(1-\lambda) y) \leq \lambda f(x)+(1-\lambda) f(y)-\lambda(1-\lambda) F(x-y),
$$

for each $\lambda \in[0,1]$ and $x, y \in I$. (Very recently, this approach has been investigated by Adamek in $[1])$. 
We should note that, if $F$ is $F$-strongly affine (i.e. "=" instead of " $\leq$ " in (4.1)) then the function $f$ is $F$-strongly convex if and only if $g=f-F$ is convex (see [1, Lemma 4]).

From (4.1) we infer that

$$
f(\lambda(x-y)+y)-f(y)+\lambda(1-\lambda) F(x-y) \leq \lambda(f(x)-f(y)) .
$$

By dividing both sides by $\lambda$ we obtain

$$
\frac{f(\lambda(x-y)+y)-f(y)}{\lambda}+(1-\lambda) F(x-y) \leq f(x)-f(y)
$$

Notice that if $f$ is differentiable, then by letting $\lambda \rightarrow 0$ we find that

$$
f^{\prime}(y)(x-y)+F(x-y)+f(y) \leq f(x)
$$

for all $x, y \in I$ and $\lambda \in[0,1]$.

In a manner similar to the proof of Theorem 3.3, if $F$ is a continuous function it follows from (4.2) that

$$
f(\langle A x, x\rangle) \leq\langle f(A) x, x\rangle-\langle F(A-\langle A x, x\rangle) x, x\rangle,
$$

for any self-adjoint operator $A$ and $x \in \mathcal{H}$, with $\|x\|=1$.

Inequality (4.3) in a weaker form was obtained by Kian in [5, Theorem 2.1] for superquadratic functions.

The following theorem is a generalization of (4.3). The idea of the proof, given below for completion, is similar to that in [8, Lemma 2.3].

Theorem 4.1. Let $f: I \rightarrow \mathbb{R}$ be an F-strongly convex and differentiable function on $\stackrel{o}{I}$ and let $F: I \rightarrow[0, \infty)$ be a continuous function. If $A$ is a self-adjoint operator on the Hilbert space $\mathcal{H}$ with $\operatorname{Sp}(A) \subset \stackrel{o}{I}$ and $f(0) \leq 0$, then

$f(\langle A x, x\rangle) \leq\langle f(A) x, x\rangle-\left\langle F\left(A-\frac{1}{\|x\|^{2}}\langle A x, x\rangle\right) x, x\right\rangle+\left(\|x\|^{4}-\|x\|^{2}\right) F\left(\frac{1}{\|x\|^{2}}\langle A x, x\rangle\right)$

for each $x \in \mathcal{H}$ and $\|x\| \leq 1$. 
Proof. Let $y=\frac{x}{\|x\|}$ so that $\|y\|=1$. Whence

$$
\begin{aligned}
f(\langle A x, x\rangle)= & f\left(\|x\|^{2}\langle A y, y\rangle+\left(1-\|x\|^{2}\right) 0\right) \\
\leq & \|x\|^{2} f(\langle A y, y\rangle)+\left(1-\|x\|^{2}\right) f(0)-\|x\|^{2}\left(1-\|x\|^{2}\right) F(\langle A y, y\rangle-0) \quad(\text { by }(4.1)) \\
\leq & \|x\|^{2} f(\langle A y, y\rangle)+\left(\|x\|^{4}-\|x\|^{2}\right) F(\langle A y, y\rangle) \quad(\text { since } f(0) \leq 0) \\
\leq & \|x\|^{2}(\langle f(A) y, y\rangle-\langle F(A-\langle A y, y\rangle) y, y\rangle)+\left(\|x\|^{4}-\|x\|^{2}\right) F(\langle A y, y\rangle) \quad(\text { by }(4.3)) \\
= & \|x\|^{2}\left(\frac{1}{\|x\|^{2}}\langle f(A) x, x\rangle-\frac{1}{\|x\|^{2}}\left\langle F\left(A-\frac{1}{\|x\|^{2}}\langle A x, x\rangle\right) x, x\right\rangle\right) \\
& +\left(\|x\|^{4}-\|x\|^{2}\right) F\left(\frac{1}{\|x\|^{2}}\langle A x, x\rangle\right) \\
= & \langle f(A) x, x\rangle-\left\langle F\left(A-\frac{1}{\|x\|^{2}}\langle A x, x\rangle\right) x, x\right\rangle+\left(\|x\|^{4}-\|x\|^{2}\right) F\left(\frac{1}{\|x\|^{2}}\langle A x, x\rangle\right) .
\end{aligned}
$$

This completes the proof.

Remark 4.1. By taking into account that $F(\cdot)$ is a non-negative function, Theorem 4.1 provides an improvement for [8, Lemma 2.3]. More precisely we have

$$
\begin{aligned}
f(\langle A x, x\rangle) & \leq\langle f(A) x, x\rangle-\left\langle F\left(A-\frac{1}{\|x\|^{2}}\langle A x, x\rangle\right) x, x\right\rangle+\left(\|x\|^{4}-\|x\|^{2}\right) F\left(\frac{1}{\|x\|^{2}}\langle A x, x\rangle\right) \\
& \leq\langle f(A) x, x\rangle .
\end{aligned}
$$

\section{Acknowledgments}

The authors express their gratitude to the anonymous referees for their careful reading and detailed comments which have considerably improved the paper.

\section{REFERENCES}

[1] M. Adamek, On a problem connected with strongly convex functions, Math. Inequal. Appl. 19(4) (2016), $1287-1293$.

[2] S.S. Dragomir, Bounds for the normalized Jensen functional, Bull. Austral. Math. Soc. 74(3) (2006), 471476.

[3] J.B. Hiriart-Urruty, C. Lemaréchal, Fundamentals of Convex Analysis, Springer, Berlin (2001).

[4] S. Ivelić, A. Matković, J. Pečarić, On a Jensen-Mercer operator inequality, Banach J. Math. Anal. 5(1) (2011), 19-28.

[5] M. Kian, Operator Jensen inequality for superquadratic functions, Linear Algebra Appl. 456 (2014), 82-87.

[6] W. Liao, J. Wu, J. Zhao, New versions of reverse Young and Heinz mean inequalities with the Kantorovich constant, Taiwanese J. Math. 19(2) (2015), 467-479.

[7] A. Matković, J. Pečarić, I. Perić, A variant of Jensen's inequality of Mercer's type for operators with applications, Linear Algebra Appl. 418(2) (2006), 551-564.

[8] J.S. Matharu, M.S. Moslehian, J.S. Aujla, Eigenvalue extensions of Bohr's inequality, Linear Algebra Appl. 435(2) (2011), 270-276. 
[9] C.A. McCarthy, $c_{p}$, Israel J. Math. 5 (1967), 249-271.

[10] A.McD. Mercer, A monotonicity property of power means, J. Ineq. Pure and Appl. Math. 3(3) (2002), Article 40.

[11] A.McD. Mercer, A variant of Jensen's inequality, J. Ineq. Pure and Appl. Math. 4(4) (2003), Article 73.

[12] F.-C. Mitroi, Estimating the normalized Jensen functional, J. Math. Inequal. 5(4) (2011), 507-521.

[13] F.-C. Mitroi-Symeonidis, N. Minculete, On the Jensen functional and the strong convexity, Bull. Malays. Math. Sci. Soc., (2016) DOI:10.1007/s40840-015-0293-Z.

[14] B. Mond, J. Pečarić, Convex inequalities in Hilbert space, Houston J. Math. 19 (1993), 405-420.

[15] N. Merentes, K. Nikodem, Remarks on strongly convex functions, Aequationes Math. 80(1-2) (2010), 193199.

[16] M. Nelson, K. Nikodem, S. Rivas, Remarks on strongly Wright-convex functions, Annales Polonici Mathematici. 102(3) (2011), 271-278.

[17] M. Niezgoda, A generalization of Mercer's result on convex functions, Nonlinear Anal. 71(7) (2009), 27712779.

[18] K. Nikodem, On Strongly Convex Functions and Related Classes of Functions, Handbook of Functional Equations. Springer New York (2014), 365-405.

[19] K. Nikodem, Z. Páles, Characterizations of inner product spaces by strongly convex functions, Banach J. Math. Anal. 5(1) (2011), 83-87.

[20] B.T. Polyak, Existence theorems and convergence of minimizing sequences in extremum problems with restrictions, Soviet Math. Dokl. 7 (1966), 72-75.

[21] A.W. Roberts, D.E. Varberg, Convex functions, Academic Press, New York-London (1973).

[22] S. Simić, Best possible global bounds for Jensen functional, Proc. Amer. Math. Soc. 138(7) (2010), 24572462.

[23] H. Zuo, G. Shi, M. Fujii, Refined Young inequality with Kantorovich constant, J. Math. Inequal. 5(4) (2011), $551-556$.

\footnotetext{
${ }^{1}$ Department of Mathematics, Mashhad Branch, Islamic Azad University, Mashhad, Iran.

E-mail address: hrmoradi@mshdiau.ac.ir

${ }^{2}$ Department of Mathematics, Mashhad Branch, Islamic Azad University, Mashhad, Iran.

E-mail address: erfanian@mshdiau.ac.ir

${ }^{3}$ Department of Mathematics, University of Peshawar, Peshawar, Pakistan.

E-mail address: adilswati@gmail.com

${ }^{4}$ Department of Mathematics, University of Bielsko-Biala, Ul. Willowa 2, 43-309 Bielsko-Biala, Poland.

E-mail address: knikodem@ath.bielsko.pl
} 